Les Rayons $\alpha, \beta, \gamma$ des Corps Radioactifs en Relation avec la Structure Nucléaire. By Madame P. Curie. (Actualités Scientifiques et Industrielles, No. 62.) Paris, Hermann, 1933. 40 pp.

In this monograph, which is quite non-mathematical, the famous discoverer of radium, whose recent death was a great loss to science, discusses the recent attempts to obtain information about the nature of the nuclei of atoms from a study of the inhomogeneities present in their radiations. Reference is made to the complexities introduced by the recent conceptions of neutrons and positrons. The experimental evidence seems to support Gamow's hypothesis that the nucleus, like Bohr's atom, possesses energy levels, $\alpha$-particles playing the role of electrons.

F. D. Murnaghan

Vorlesungen ïber Projektive Geometrie. By C. Juel. Berlin, Springer, 1934. $\mathrm{xi}+287 \mathrm{pp}$.

C. Juel, who is professor emeritus at the Technical High School of Kopenhagen, has written a book on projective geometry with special consideration of von Staudt's theory of imaginaries.

He assumes a knowledge of ordinary real projective geometry as for example contained in F. Enriques' Vorlesungen über projektive Geometrie. Professor Juel has succeeded in giving as clear a treatment as possible of an otherwise cumbersome and somewhat obsolete method. At the present time one is inclined to make use of the much more effective and elegant analyticgeometric method with its great time-saving factor.

Among the particular features of the book may be mentioned chapters on the plane cubic curve and the quadratic transformation.

ARnold EMCH

Theorie der Funktionen Mehrerer Komplexen Veränderlichen. By H. Behnke und

P. Thullen. Ergebnisse der Mathematik und ihrer Grenzgebiete, Volume 3.

Berlin, J. Springer, 1934. $115 \mathrm{pp}$.

This little book surveys in a compact and scholarly fashion the present status of some basic questions in the yet immature but rapidly developing subject of functions of several complex variables. The authors acknowledge their initial indebtedness to Osgood's Lehrbuch der Funktionentheorie, II, 1: Grundlagen der allgemeinen Theorie der Funktionen Mehrerer Komplexen Grössen, 1924, comparison with which gives evidence of the progress achieved during the last decade.

The chapter headings suggest the scope of this work: I. Domains over the extended space. II. Geometrical foundations. III. Representation of regular functions by elementary series. IV. Singular manifolds. V. Distribution of zeros and of nonessential singularities. VI. Theory of domains of regularity and of shells of regularity (Regularitätshüllen). VII. Transformation theory. Much of this work is of necessity occupied with extending familiar concepts in an obvious way either from one variable to $n$, or from real geometry to complex. The authors contrast two traditional types of $n$-space, one called by Osgood the "space of analysis," and having $n$ hyperplanes at infinity, and the other the projective space having a single hyperplane at infinity. Instead of 
retaining throughout both alternatives, the projective space is here adopted as basis for discussion. Significant early concepts that may prove new to the reader include those of pseudo-convexity, cylinder-domain, and a surface of determination for it, dicylinder, Reinhardt domain (of which hyperspheres and dicylinders are examples), circular domain, Hartogs domain (having regard to a single variable), Cartan domain.

The sixth chapter elaborates the implications of the following striking theorem (page 39): "Every function $f(w, z)$ regular in a proper Reinhardt domain $K$ is developable in a power series convergent throughout $K$ and is furthermore analytically extensible throughout the interior of the minimal complete Reinhardt domain containing $K$." The final chapter discusses the automorphic mapping of a region particularly with respect to the invariance of the center. Use is made of the metric of Carathéodory in which distance between two points, $A$ and $B$, is given by the upper bound of

$$
\log \frac{|f(A)-f(B)|+|1-f(A) \overline{f(B)}|}{[(1-f(A) \overline{f(A)})(1-f(B) \overline{f(B)})]^{1 / 2}}
$$

with respect to all permissible functions, $f$.

It is clear that the subject is acquiring a content other than as a simple hybrid product of the theory of one complex variable crossed with the geometry of the real plane. One notes, however, as marking presumably a transient aspect, that the results here given are fairly illustrated by the case of two variables, theorems for higher dimensions being as yet limited to relatively trivial generalizations from the important case of $n=2$. The postulational approach is avoided in this work so that questions of abstract space and even the tractable case of Hilbert space are not suggested although in so far as the particular value of $n$ remains non-significant some of the theory might be expected to depend upon features available under more general conditions.

In generalizing from one variable it is natural to take up first the case of a single function of $n$ independent complex variables, dealing with series expansions, singularities, regions of convergence, and like questions. Problems relating to uniform mapping and to invariants under analytic transformations, when generalized to $n$ variables, suggest the study of simultaneous systems of $n$ functions of $n$ variables, as emphasized by Forsyth in the earliest book on the subject, Lectures Introductory to the Theory of Functions of Two Complex Variables, 1914. As remarked above, the last chapter of the present work gives some recent results in this direction, but not in the vectorial language symmetry would seem to demand. Indeed one may feel that despite some progress toward a geometrical viewpoint, the independent variables have remained a trifle too obstinately independent in the present theory and have not been completely merged into the concept of a point in a homogeneous projective space for which a choice of coordinates is extraneous or adventitious.

But whether or not one may have the impression that this discussion of the subject calls for a more independent abstract treatment and broader vision, the reader will not fail to appreciate the concrete details here arranged in logical order with abundant references to the literature and the inclusion of explanatory and illustrative material where most needed.

\section{A. A. Bennett}

